\section{Cahiers de Narratologie}

Analyse et théorie narratives

$10.1 \mid 2001$

La voix narrative

\title{
El crimen de la Cuenca de Pilar Mirò : des récits factuels au récit fictif
}

\section{Catherine Berthet-Cahuzac}

\section{(2) OpenEdition \\ 1 Journals}

\section{Electronic version}

URL: http://journals.openedition.org/narratologie/6931

DOI: 10.4000/narratologie.6931

ISSN: 1765-307X

\section{Publisher}

LIRCES

\section{Printed version}

Date of publication: 1 January 2001

Number of pages: 149-157

ISBN: 2914561032

ISSN: 0993-8516

\section{Electronic reference}

Catherine Berthet-Cahuzac, "El crimen de la Cuenca de Pilar Mirò : des récits factuels au récit fictif", Cahiers de Narratologie [Online], 10.1 | 2001, Online since 20 October 2014, connection on 23 February 2021. URL: http://journals.openedition.org/narratologie/6931 ; DOI: https://doi.org/10.4000/ narratologie.6931 


\title{
EL CRIMEN DE CUENCA DE PILAR MIRÓ : DES RÉCITS FACTUELS AU RÉCIT FICTIF
}

\author{
Catherine BERTHET-CAHUZAC \\ Université de Montpellier III
}

El crimen de Cuenca, film réalisé par Pilar Miró en 1979 et sorti sur les écrans en 1980, démonte avec minutie le mécanisme d'une erreur judiciaire qui fut à l'origine d'un scandale de portée nationale. Le scénario, solidement documenté, a été rédigé après une enquête sur le terrain de la co-scénariste Salvador Maldonado, partie travailler sur les archives du Tribunal de Cuenca et recueillir les témoignages des survivants de l'affaire'. Le film, qui adopte la forme de la reconstitution historique, prend explicitement appui sur un faisceau particulièrement étoffé de textes préexistants (pièces d'instruction, plaidoiries et réquisitoires, témoignages des descendants des inculpés, articles de presse parus lorsque la vérité s'est fait jour, arrêt de la Cour de Cassation annulant le jugement premier et aussi récits fictifs brodés autour de cet événement). L'effet de polyphonie qui s'ensuit est amplifié par l'injection du film lui-même dans le contexte judiciaire contemporain, puisque El crimen de Cuenca a fait l'objet de poursuites. Le tribunal n'a autorisé la sortie sur les écrans d'une œuvre suspectée de porter atteinte aux grands corps de l'État qu'avec l'adjonction d'un carton préliminaire rédigé par ses soins. Réalisatrice et producteur ont pu à juste titre se réjouir de l'obligation qui leur était faite : le jeu de miroir entre récit factuel et récit fictif se trouvait ainsi reproduit en aval du film.

1 Voir Salvador MALDONADO, El crimen de Cuenca, Barcelona, Ed. Argos Vergara, 1980. 
En 1913, deux paysans de la province de Cuenca ont été inculpés, malgré l'absence de faits tangibles, du meurtre d'un compagnon de travail, disparu sans laisser de trace trois ans plus tôt. La procédure engagée à leur encontre a été des plus chaotiques. Une première décision de non lieu a été suivie de la réouverture des mois plus tard du dossier, sur la base de simples rumeurs. L'instruction a été menée dans la plus grande partialité (les illégalités de procédure, les pressions sur témoins, l'extorsion d'aveux sous la torture ont été dénoncés dans l'arrêt de la Cour de Cassation, en 1926). L'ouverture du procès s'est trouvée longtemps différée en raison des compléments d'information régulièrement demandés par un avocat général fort réticent face aux carences manifestes de l'instruction - mais concluant pourtant au bout de plusieurs années en demandant la peine de mort. Obligés de plaider coupable pour faire valoir de prétendues circonstances atténuantes, les inculpés ont purgé leur peine avant que la supposée victime, pourtant installée dans un village guère éloigné du lieu de l'affaire, ne redonne signe de vie.

Dans le film de Pilar Miró, ces événements servent de prétexte à une critique acerbe du caciquisme en vigueur au début du siècle. El crimen de Cuenca donne à voir le dévoiement de la machine judiciaire, mise au service du politique. Mais faut-il s'étonner de ce qu'en parlant du passé, on parle aussi de soi et de son présent ? Le concept de manipulation s'autonomise. Débordant le simple prétexte historique, le film met en cause la distorsion progressive des données de départ ou, si l'on préfère, s'interroge sur le phénomène de réécriture d'un événement déterminé.

La polyphonie énonciative de fait se trouve reflétée dans la dualité formelle de El crimen de Cuenca. Dans un précédent article, j'ai montré comment le narrateur, omniscient, se prévaut de modalités de récit contrastées pour faire surgir la duplicité des discours cités. Un récit dépouillé où le narrateur simule une attitude neutre alterne avec un récit chargé d'affect, qui emprunte au mélodrame ou au sensationnalisme. Les scènes de torture difficilement soutenables ont fait la célébrité du film, mais elles ne restent à mon sens compréhensibles qu'en regard de cette autre modalité narrative, d'une grande sobriété. Pour moi, la mise en perspective 
de formes de récit divergentes signe une distanciation du narrateur par rapport à son propre énoncé, rend perceptible l'imitation critique des récits premiers (le récit d'aveugle, mais aussi le réquisitoire et les plaidoiries, traversés eux-mêmes par la dialectique entre affectation d'objectivité et sensationnalisme).

C'est en ce sens que j'avais parlé de retournement des discours cités, oppressifs et/ ou aliénants. Le dialogisme met en évidence une circulation du sens perverse entre des énoncés censés être étanches. Choix des fragments textuels reproduits (plaidoirie, réquisitoire, copla) et dialogues fictifs présentant des moments définitivement inaccessibles à l'historien entrent en système en démultipliant l'effet de va-et-vient entre la rumeur publique et l'appareil judiciaire, celui-ci étant mu par / et alimentant celle-là. Je prendrai l'exemple de la visite de Dolores à son mari incarcéré. Si l'entrevue des deux personnages et l'altercation qui s'est ensuivie sont attestées, on ne saurait par contre connaître la teneur exacte des propos échangés en tête à tête. Le dialogue recréé pointe la responsabilité des autorités judiciaires à l'origine de l'affabulation (Diré lo que él [le juge Isasa] quiere). D'autre part, la continuité filmique, qui bouscule sensiblement la chronologie des faits à ce moment du récit, place les conclusions provisoires de l'avocat général (citées littéralement) en moyen terme entre ces aveux provoqués et la copla colportée par l'aveugle de village en village.

En somme, le fictionnel sert de réactif au factuel, qui s'avère être lui-même une forme fictionnalisante puisque les conclusions de l'avocat général consistent en un descriptif détaillé d'un meurtre qui n'a jamais eu lieu². La contradiction est forte, l'affabulation étant le résultat d'un discours qui a pour vocation d'être un acte de dévoilement. C'est la frontière entre fictif et factuel qui est en cause. Le choc des discours pose le problème de la véracité et/ ou de la vraisemblance de l'asserté.

2 Dans un article précédent, j'avais relevé les points de coïncidence entre les récits inauthentiques (aveux de Dolores/ copla / rumeur publique) et les conclusions du procureur (dans actes du Colloque "Univers répressifs dans le monde hispanique et lusophone", Univ. Nancy II mai 1999, à paraître). 
Dès l'incipit, l'instance d'énonciation se place dans son rapport aux narrateurs cités (réels ou fictifs). L'arrêt sur image concomitant du générique initial coupe littéralement la parole au narrateur délégué (l'aveugle mis en scène au prégénérique), qu'il fige, la bouche grande ouverte, au beau milieu de son récit. Le passage au générique instaure donc un rapport d'autorité entre instance citante et instance citée. La première se donne à voir comme l'instance qualifiante qui légitime ou discrédite les différents énoncés convoqués dans le film. En adoptant la forme de la reconstitution historique, l'énonciateur se place en position de commentateur omniscient. La sobriété d'une grande partie du récit a valeur véridictoire. Cartons et plans de coupe attestent d'une référenciation précise, du travail préalable de documentation. L'utilisation réitérée des plans de coupe (vues en ext.jour des lieux de l'action) et de cartons toujours sur le même modèle (mention du lieu et de la date précise) produit un effet de monotonie qui peut se lire comme un refus affiché de fictionnalisation. Le narrateur feint de présenter un exposé sommaire des faits en respectant les trois requis de la narratio rhétorique, brièveté, clarté, vraisemblance. Le texte filmique semble ne conserver que les éléments nécessaires à la bonne compréhension des faits. Les indications temporelles données par les cartons ou inscrites dans les fragments textuels cités (mandat d'amener / mandat d'arrêt puis acte d'accusation) rendent compte d'une chronologie rigoureuse, d'un ordre du récit apparemment conforme à l'ordre des événements.

Mais cependant des écarts sont perceptibles, dès lors que l'on confronte fiction et données historiques. Les cartons introduisent une certaine duplicité puisque les datations sont parfois mensongères. Le carton de la première séquence (Cuenca manchega, 28 de agosto, 1910) réfère à une péripétie survenue en août 1911. Les conclusions provisoires de l'avocat général (lues en voix off) datent du 25/8/1915 et non, comme indiqué, du 25/5/1918 (date, celle-là, du procès et de la lecture du réquisitoire définitif).

La chronologie des événements est ainsi remaniée de façon à introduire de nouveaux liens de causalité. C'est ce qui permet notamment de donner à la mort de don Francisco (maire de Osa de la Vega et employeur, au moment des faits 
incriminés, des deux accusés) un caractère décisif L'agencement du texte filmique (délivrance de l'extrême onction/ violation du secret de la confession par le député Contreras, qui écoute à la porte $>$ fondu au noir ponctuant une première étape $>$ visite de Contreras et du curé au nouveau juge, récemment nommé, dont ils font ainsi la connaissance) laisse entendre que la confession accusatrice est à l'origine de la réouverture du dossier. Dans la réalité, il s'agit d'un élément parmi d'autres alimentant une rumeur publique diffuse, dont on ne peut à coup sûr cerner une source unique. Le juge Isasa a été nommé en remplacement du juge Rodriguez et a rencontré Contreras et le curé don Rufo plusieurs mois avant le décès du notable. Les événements, il est vrai, se précipitent dans les semaines qui suivent, jusqu'à provoquer la réouverture du dossier d'instruction. Mais celle-ci intervient à la suite de la visite d'une commission d'enquête envoyée sur la requête de la famille. Ce seul exemple suffit à montrer comment le narrateur reconstruit un nouveau continuum, "lisse » une série d'événements qu'il articule autour de sa démonstration, ouvertement manichéenne. Don Rufé et, bien plus encore, le député Contreras, sont les principales cibles de la critique. Le film brode littéralement autour des deux personnages, pour forcer le trait. S'il est vrai que le prêtre a quelque peu tardé à faire état de la lettre émanant du « disparu » et en a référé d'abord à Contreras, la manigance n'est pas pour autant patente.

Le propos du film, qui vise à dénoncer le fonctionnement oligarchique d'une société régie par la collusion des pouvoirs, suffirait à lui seul à poser la question de la véracité de l'énoncé. El crimen de Cuenca prétend démasquer les instigateurs de la machination, lever les zones d'ombre laissées par l'Histoire. On voit bien, à lire l'ouvrage de Salvador Maldonado, qu'on ne peut guère trouver de traces matérielles de l'ingérence du politique (et plus particulièrement, de Contreras). La scénariste en est réduite à recouper des faits dont la concomitance est troublante et monter à partir de là des hypothèses, au demeurant fort plausibles. La mutation du juge Rodriguez à la suite de la décision de non lieu, la démission du greffier fort diligent, entré au service de Contreras, soulèvent effectivement bien des interrogations. 
Mais les plans rapprochés sur des regards entendus transforment l'hypothétique en assertif. S'il en simule à certains moments la forme, le film ne se veut pas pour autant une reconstitution historique. La vraisemblance prend le pas sur l'exactitude du compte-rendu tout en se montrant au service d'une vérité d'une autre nature. Il faut ajouter à cela que les distorsions par rapport au réel ne peuvent s'expliquer par la seule nécessité de condenser le récit filmique. Pourquoi sinon toujours représenter Dolores avec son bébé dans les bras en faisant fi du temps qui court (de 1911 à 1915, voire 1918 !) si ce n'est pour susciter immédiatement un consensus autour de la figure stéréotypée de la Mère à l'enfant, placée dans le film en position de victime innocente ? Il est clair que la finalité persuasive du discours l'emporte sur la vraisemblance elle-même.

Le concept de manipulation, dont je rappelais précédemment qu'il est fondamental dans le dispositif sémiotique du film, affecte l'instance d'énonciation elle-même. Car celle-ci se révèle dans sa dualité, dans la mesure où elle tend à concilier formes objectivantes et position polémique. Les cartons sont au cœur de cette stratégie. El Crimen de Cuenca se prévaut de leur statut ambigu. Ils relèvent en effet d'un niveau sémiotique spécifique et se distinguent donc du récit iconico-verbal sur lequel ils sont surimposés.

De sorte qu'ils fonctionnent à la fois en tant que composante du texte filmique et paratexte. Ils contribuent à poser l'énonciateur en commentateur d'un énoncé ainsi distancié. Les implications sont doubles. Les cartons dans le même temps suggèrent un effacement et une intromission de l'instance d'énonciation car, s'ils sont laconiques et presque toujours dépourvus de déictiques, le texte inséré n'en est pas moins un rajout à considérer comme tel. D'autre part, le recours aux cartons tend à confondre les deux instances discursives antinomiques à l'origine, pour l'une, du texte filmique, et pour l'autre, du premier carton imposé par les censeurs. Je m'étais attachée jusqu'ici à montrer comment le deuxième carton, dédicace au village de Osa de la Vega, qui est apparu comme une victime collective de l'injustice, puis l'ensemble du film réagissent sur le premier carton en en 
faisant surgir le caractère mensonger (no hay... la menor intención ofensiva...). Il n'en reste pas moins que les modalités discursives auxquelles il est fait recours sont similaires. Hormis l'emploi de la première personne du pluriel dans le deuxième carton (Dedicamos...), les énoncés sont nettement débrayés. De surcroît, aucune marque formelle ne vient différencier le premier carton des suivants. Au contraire, un fondu au noir avant le prégénérique isole les deux premiers cartons, qui prennent ainsi l'allure d'une unité narrative symétrique du générique final accompagné lui aussi d'une série de cartons nous informant des événements survenus en après-récit.

Je retrouve là un effet d'homogénéisation semblable à celui produit par le recours à la voix off. Par trois fois, des décisions judiciaires sont proférées hors champ, par derrière un récit iconico-verbal donnant à voir les incidences de ces notifications (mandat de comparution émis par le juge Rodriguez/ mandat d'arrêt émis par le juge Isasa/ conclusions du procureur). Bien que les voix soient différentes, la variation de timbre n'est guère prononcée. Le ton, monocorde, de la lecture unifie les trois énoncés, dont les caractères particularisants sont gommés. Les trois passages semblent l'émanation d'une entité mystérieuse, agissant dans l'ombre et au pouvoir redoutable. La parole judiciaire est désincarnée (on notera d'ailleurs que le dernier plan accompagnant la lecture du réquisitoire montre une salle de tribunal déserte / la chaise de l'avocat général reste vide, pendant les scènes du procès). Cette parole apparaît comme l'émanation d'un acousmètre. Je reprends là la terminologie de $\mathrm{M}$. Chion, qui désigne ainsi les personnages acousmatiques, c'est-à-dire entendus hors champ sans être jamais visualisés ${ }^{3}$, et dont la présence toute particulière se soutient de leur absence même au sein de l'image.

C'est à ce titre que j'avais parlé d'une homologie entre les cartons et la voix off. Le film combine là deux procédés qui confèrent au narrateur concerné l'aura d'un savoir ne donnant pas prise à la discussion. On pourrait reprendre pour les cartons les remarques de $\mathrm{M}$. Chion à propos de l'acous-

${ }^{3}$ Dans La voix au cinéma, Paris, Ed. de l'Etoile, 1985, p. 109-110. 
mètre, auquel sont attribués souvent, dans les fictions cinématographiques, trois pouvoirs plus un don, premièrement le pouvoir d'omnivoyance, deuxièmement celui d'omniscience, et troisièmement la toute-puissance d'agir sur la situation. S'il est vrai que le fictif réagit sur le factuel, celui-ci reste le discours d'autorité de référence, un modèle dénoncé, certes, mais par l'imitation de certaines de ses caractéristiques ${ }^{4}$. Il faut plutôt parler d'interaction. En interrogeant le pouvoir de la parole, l'énonciateur filmique se met forcément lui-même en question.

$\mathrm{Ce}$ questionnement est double. Il serait en effet réducteur de voir dans la voix off un simple signe d'autorité. L'acousmatisation des voix est un phénomène généralisé dans le film, qui déborde le seul recours à la voix off (anticipations et retards sonores abondent). Or il s'agit là d'un procédé foncièrement ambivalent. Dans le même temps qu'elle peut instituer l'énonciateur en entité omnisciente, douée d'ubiquité, l'acousmatisation vient aussi signaler le phénomène de vissage du sonore sur le visuel, constitutif du matériau filmique. Le montage sonore et/ou musical dément à maintes reprises la segmentation nettement affirmée du montage iconique. Il nous est même donné à voir à certains moments un montage global irréductible à toute chronologie.

Comme nombre de cinéastes de l'époque, Pilar Miró revendique le caractère fictionnalisant de son œuvre. On ne doit pas y voir une contradiction avec le volontaire positionnement par rapport à des récits factuels préexistants. Dans les deux cas, nous nous trouvons face à un texte filmique qui interroge ses marges. El crimen de Cuenca s'inscrit par là dans toute une production culturelle qui pose la question de la difficile intelligibilité du réel. La posture phénoménologique dominante dans le cinéma espagnol des années 1970 se traduit par de continuels efforts de dévoilement du leurre introduit par la fiction filmique. C. Saura, J. Chávarri notamment jouaient déjà de l'inadéquation des montages sonore et iconique, pour donner à voir l'acte de manipulation constitutif de l'élaboration filmique. Mais si, très souvent, c'est la dialectique réel/ fiction qui est fondamentale, dans le cas de $E l$

${ }^{4}$ C'est ce que je montrais dans l'article cité précédemment. 
crimen de Cuenca, c'est le concept même de manipulation qui devient central. Il découle de la mise en cause des rapports entre justice et politique, question particulièrement sensible en Espagne à la fin des années 1970. Je rappellerai que le film de Pilar Miró a été réalisé peu de temps après la promulgation, obtenue à grand-peine, de l'amnistie totale et qu'il reste, bien malgré lui, une preuve de la survivance de la censure cinématographique, à l'époque. On est en droit de supposer que les circonstances du moment ont motivé la réémergence du fait divers vieux de 70 ans. L'affinité conceptuelle est en effet bien grande entre les deux notions de réhabilitation et d'amnistie. 